\title{
Changes in Electrocardiogram Findings during Treatment with Gonadotropin-Releasing Hormone Agonist and Surgical Castration for Prostate Carcinoma
}

\author{
Hasan Sağlam ${ }^{1}$ Akif Çakar², Osman Köse ${ }^{3}$, Şükrü Kumsar ${ }^{3}$, Salih Budak $^{3}$, \\ Serbülent Gökhan Beyaz ${ }^{4 *}$, Öztŭ Adsan $^{1}$ \\ ${ }^{1}$ Department of Urology, Sakarya University Medical School, Sakarya, Republic of Turkey \\ ${ }^{2}$ Department of Cardiology, Sakarya University Training and Research Hospital, Sakarya, Republic of Turkey \\ ${ }^{3}$ Department of Urology, Sakarya University Training and Research Hospital, Sakarya, Republic of Turkey \\ ${ }^{4}$ Anesthesiology and Pain Management Center, Sakarya University Medical School, Sakarya, Republic of Turkey \\ Email: ${ }^{*}$ sgbeyaz@gmail.com
}

Received July 4, 2012; revised August 6, 2012; accepted September 10, 2012

\begin{abstract}
Purpose: To investigate electrocardiogram (ECG) changes after complete androgen blockade (CAB) achieved by either surgical or medical castration and compare the outcomes of the groups. Methods: Sixty-three consecutive men (between 58 - 86 years of age) requiring $\mathrm{CAB}$ for prostate cancer were enrolled in the study. Patients with diabetes mellitus, an additional malignancy, coronary heart disease, atrial fibrillation, heart failure or a medical history of cardiac event in the last 12 months were excluded from the study. Additionally, those who were taking medicine affecting heart rate were excluded. The participants were divided into two groups according to their modality of castration. The first group consisted of 35 patients who received bilateral orchiectomy plus anti-androgen medication. The second group contained 28 patients who accepted gonadotropin-releasing hormone $(\mathrm{GnRH})$ plus anti-androgen therapy. After complete examinations and biochemical tests, the ECG leads of the patients were obtained conveniently. This was then repeated at three- and six-month visits. ECG findings (including heart rate, PR, QRS, QT, corrected QT (QTc) intervals and QT dispersion (QTd)) were recorded and analysed statistically. The groups were then compared in terms of pre- and post-treatment ECG outcomes. Results: Both groups revealed similarly lower heart rate and prolonged PR, QRS, QT, corrected QTc and QTd by the end of six months. By the end of three months, all variables had changed significantly in the orchiectomy group, whereas in the GnRH group, they had not. Conclusion: CAB may result in lower heart rate and prolonged QT, a condition associated with fatal cardiac arrhythmia and sudden death. Therefore, patients receiving $\mathrm{CAB}$ should be monitored closely for cardiac adverse effects.
\end{abstract}

Keywords: Gonadotropin-Releasing Hormone; Castration; Testosterone; Electrocardiogram; QT Interval; Heart Rate

\section{Introduction}

Prostate cancer has been shown to be the second most common malignancy and sixth leading cause of death in men worldwide. Epidemiological studies reveal that its incidence is on the rise in the era of prostate-specific antigen (PSA) $[1,2]$. In contrast to localised prostate cancer for which many therapeutic modalities exist (including radiotherapy, surgery, chemotherapy etc.), in metastatic prostate cancer, hormonal therapy (i.e. androgen deprivation therapy (ADT)) is the treatment of choice as an initial therapy. ADT is achieved by either surgical removal of the bilateral testes or medical therapy with gonadotropin releasing-hormone $(\mathrm{GnRH})$ analogues or antagonists. It has traditionally been a cornerstone in

"Corresponding author. the treatment of advanced or metastatic prostate cancer, and it has been effectively used for two to three years on average [3]. If such a deprivation therapy is used in conjunction with an androgen receptor antagonist to block the effects of androgens of adrenal origin, it is termed a combined androgen blockade (CAB) [4].

Hormonal therapy is also being used for localised prostate cancer in high-risk patients. Therefore, the use of ADT in the earlier and later stages of prostate cancer has increased over time, and consequently, more men are being treated with ADT for longer periods [5]. Cardiovascular disease (CVD) has recently been investigated as one of the adverse effects of ADT, which may be due to interference with the cardioprotective effect of testosterone. Despite male gender is a risk factor for CVD, data 
are increasing on cardioprotection of testosteron. ADT has been shown to increase approximately $10 \%$ of adiposity and cholesterol increase which are risk factors for CVD. Hypogonadism has been found to be associated with hyperinsulinemia which may elevate cardiac risk through a link to diabetes. Lower serum testosterone has also been associated with a prothrombotic state, which may predispose men to myocardial infarction. Studies of animal models of atherosclerosis have demonstrated that after castration, aortic atherosclerosis was increased $[4,6,7]$.

In this study, in addition to above mentioned effects of ADT on the heart, we have aimed to investigate the effects of $\mathrm{CAB}$ on ECG measures that might be suggestive of cardiac adverse effects and compare the results of the group received GnRH plus anti-androgen therapy with those of the group received bilateral orchiectomy plus anti-androgen therapy.

\section{Materials and Methods}

Between May 2010 and January 2012, a total of 63 consecutive patients who received CAB, aged between 58 and 86 years (mean $76 \pm 8$ ), were enrolled in this study. Patients with diabetes mellitus, an additional malignancy, coronary heart disease, atrial fibrillation, heart failure or a medical history of cardiac event in the last 12 months were excluded from the study. Additionally, those who were taking medicine affecting heart rate (e.g. calcium channel blockers, beta-blockers or statins) were excluded. Before castration, complete routine biochemical tests, hormone analyses and ECGs were obtained. The patients were assigned to one of two groups: 1) patients who received bilateral orchiectomy + anti-androgen therapy (bicalutamide $50 \mathrm{mg}, 1 \times 1$ ) or 2) patients who received $\mathrm{GnRH}$ analogue + anti-androgen therapy (bicalutamide $50 \mathrm{mg}, 1 \times 1)$. Orchiectomies of the 31 patients were carried out concomitantly with transurethral resection of the prostate (TURP), and four other patients underwent an orchiectomy only. In the 31 patients, orchiectomies and TURP were carried out under spinal anaesthesia with $2 \mathrm{cc}$ of $1 \%$ prilocaine solution. The other four patients underwent orchiectomy under local anaesthesia with $1 \%$ prilocaine solution. Thirty-five patients in the orchiectomy group were started on oral anti-androgen therapy just after the operation. The other group of patients was given anti-androgen therapy one week before the $\mathrm{GnRH}$ started (goserelin $10.8 \mathrm{mg}, \mathrm{sc}, \mathrm{n}=8$; leuprolide acetate $11.25 \mathrm{mg}$, sc, $\mathrm{n}=10$; and leuprolide acetate $22.5 \mathrm{mg}$, sc, $n=9)$. Three and six months after initiation of CAB, the patients were re-examined, and the previous analyses were performed again. ECGs were analysed with respect to heart rate, PR, QRS, QT, QTc intervals and QTd by the same cardiologist. The results were recorded and evaluated statistically. The data of the pre- and posttreatment conditions were compared within groups and between groups using the paired t-test and the student's t-test, respectively, with a p-value of $<0.05$ being considered significant.

\section{Results}

Before the treatment, the two groups were similar with respect to all variables, including age and ECG derivation interval outcomes $(p>0.05)$ (Table 1).

Three months later, heart rate decreased from the pretreatment value of $75 \pm 10$ beats per minute $(\mathrm{b} / \mathrm{m})$ to $72 \pm$ $6 \mathrm{~b} / \mathrm{m}(\mathrm{p}=0.017)$ and from $76 \pm 8 \mathrm{~b} / \mathrm{m}$ to $68 \pm 5 \mathrm{~b} / \mathrm{m}(\mathrm{p}=$ 0.001 ) in the medical and surgical castration groups, respectively. Pre-treatment QTc changed from $412 \pm 27 \mathrm{~ms}$ to $422 \pm 40 \mathrm{~ms}(\mathrm{p}=0.49)$ and $422 \pm 29 \mathrm{~ms}$ to $440 \pm 34$ $\mathrm{ms}(\mathrm{p}=0.007)$ in the medical and surgical groups, respectively. In the same period, QTd increased from $29 \pm$ $6 \mathrm{~ms}$ to $33 \pm 7 \mathrm{~ms}(\mathrm{p}=0.001)$ in the medical group and from $29 \pm 6 \mathrm{~ms}$ to $41 \pm 8 \mathrm{~ms}(\mathrm{p}=0.001)$ in the surgical group. Findings at the end of three and six months and comparisons between the groups are presented in Table 2.

Table 1. Pretreatment characteristics of the patients.

\begin{tabular}{cccc}
\hline & Surgical group & Medical group & \\
\hline & BO + antiandrogen & GnRH + antiandrogen & $\mathrm{p}$ \\
$\mathrm{n}$ & 35 & 28 & - \\
Age (years) & $77 \pm 8$ & $74 \pm 8$ & $\mathrm{NS}$ \\
RR (b/m) & $76 \pm 8$ & $75 \pm 10$ & NS \\
Qtc $(\mathrm{ms})$ & $422 \pm 29$ & $412 \pm 27$ & $\mathrm{NS}$ \\
\hline
\end{tabular}

BO: bilateral orchiectomy, n: number of the patients, NS: nonsignificant, $\mathrm{b} / \mathrm{m}$ : beats per minute, $\mathrm{ms}$ : milisecond.

Table 2. ECG interval values three and six months after initiation of CAB.

\begin{tabular}{lccccccc}
\hline & \multicolumn{3}{c}{3 months later } & & \multicolumn{3}{c}{ 6 months later } \\
\hline Interval & $\begin{array}{c}\text { Medical } \\
\text { treatment }\end{array}$ & $\begin{array}{c}\text { Surgical } \\
\text { treatment }\end{array}$ & p & $\begin{array}{c}\text { Medical } \\
\text { treatment treatment }\end{array}$ & Surgical \\
\hline RR (b/m) & $72 \pm 6$ & $68 \pm 5$ & 0.011 & $67 \pm 6$ & $65 \pm 5$ & NS \\
PR (ms) & $174 \pm 14$ & $175 \pm 15$ & NS & $178 \pm 11$ & $180 \pm 11$ & NS \\
QRS (ms) & $94 \pm 14$ & $96 \pm 14$ & NS & $104 \pm 15$ & $100 \pm 15$ & NS \\
QT (ms) & $394 \pm 29$ & $398 \pm 32$ & NS & $415 \pm 19$ & $414 \pm 34$ & NS \\
QTc (ms) & $422 \pm 40$ & $440 \pm 33$ & 0.055 & $434 \pm 41$ & $448 \pm 34$ & NS \\
QTd (ms) & $33 \pm 7$ & $41 \pm 8$ & 0.001 & $43 \pm 8$ & $46 \pm 8$ & NS \\
\hline
\end{tabular}

$\mathrm{b} / \mathrm{m}$ : beats per minute, NS: not significant: $\mathrm{p}>0.05$. 


\section{Discussion}

In this study, we have demonstrated that cardiac consequences of CAB reflected by ECG were markedly evident six months after the onset of therapy for all patients. As with heart rate, all variables detectable on ECG were changed significantly. QT and QTc increased after CAB, but as has been stated by Yang, it was a major challenge to specifically determine the association between sex steroid hormones and arrhythmia susceptibility, since the cardiac system is highly complex. The specific effect ought to be studied in isolation (i.e. without other contributing factors) [8].

Among the androgens, testosterone in particular is widely believed to be associated with a higher risk of CVD in men [9]. This is primarily suggested to be due to androgen abuse, which has been associated with fatal consequences, including myocardial infarction, hypertension, arrhythmia, cardiac failure and sudden death [10, 11]. The effect of testosterone on CVD in men has never been investigated in a clinical, double-blind, placebocontrolled study. In contrast, it is proposed that natural androgens in general have a preventive effect in men with regard to CVD, similar to that of oestrogens in women [12].

Conflicting experimental and clinical data exist regarding the effects of sex steroids, especially testosterone and oestrogen, on the hearts of both genders. In animal models, the physiological levels of androgens have been stated to be positively related with parasympathetic activity. In contrast, decreased androgen levels in aging males have been stated to have controversial effects on autonomic function. Regarding the autonomic control of the heart, adrenal androgens have been proposed as being crucial. However, data differs according to a number of factors, including subject type (i.e. human or animal), gender, hormones studied, hormone dose, duration of exposition, concomitant pathology etc. $[9,13,14]$.

Although being male is a risk factor for coronary artery disease, increasing evidence suggests that testosterone may actually have a cardioprotective effect in men [7]. Experimental animal studies also provide evidence that testosterone has a cardioprotective effect $[15,16]$. Keating et al. reported that men receiving ADT had a $25 \%$ higher risk of incident coronary artery disease than eugonadal men [17]. It has been assumed that ADT strongly inhibits not only the hormonal modulation of prostate cancer cells but also that of the cardiac ventricular myocytes. It has been suggested that this therapy affects ventricular repolarisation, including increasing QT intervals [18] and ventricular arrhythmias [19].

The autonomic system has been proposed to be involved in human sex hormone status. Physiologic testosterone and dehydroepiandrosterone sulphate levels have been found to be positively associated with parasympa- thetic and negatively correlated with sympathetic activities. Serum oestradiol levels have also been stated to be negatively correlated with parasympathetic and positively correlated with sympathetic activities [13]. Although the findings of our study seem contrary to this explanation, the patients we studied were beyond the physiological status after $\mathrm{CAB}$. Other studies also seem to report opposite findings [20,21], but regarding the abovementioned studies, they did not exactly conduct their work by denoting heart rate variability in men with $\mathrm{CAB}$. We have shown that heart rate decreased markedly after $\mathrm{CAB}$ achieved medically or surgically.

Although statistical analyses showed great similarities between the groups by the end of three months in terms of PR, QRS and QT, values of the surgical group were significantly different in terms of RR, QTc and QTd intervals. These earlier significant changes in the surgical group may have been due to an acute onset of a fall in testosterone and oestradiol. By the end of six months, both groups revealed significantly lowered heart rate and increased PR, QRS, QT, QTc and QTd measures.

Noord et al. concluded that lower QTc intervals in men were associated with higher male sex hormones [22]. In accordance with this study, in our patients, CAB was shown to be related with longer QT and QTc intervals. On the other hand, others have stated that the temporal effects of low testosterone levels were related with shorter QT intervals in men with primary and secondary hypogonadism as well as in experimental animal observations following orchiectomy $[23,24]$.

Being a retrospective study and having a small number of patients may be first prominent limitations of this study with respect to reaching an exact conclusion. Another issue may be that the medical castration group was heterogeneous due to the fact that three similar but different medications were administered. Additionally, information about daily physical activity changes, smoking and other factors comprising metabolic syndrome was not collected completely. A general challenge that should obviously also be addressed is the difficulty in calculating QT intervals accurately.

In conclusion, $\mathrm{CAB}$, with either medical or surgical castration of men with prostate cancer, results in similar ECG changes six months after the initiation of therapy. Surgical castration reveals outcomes earlier than medical castration in terms of heart rate, QTC and QTd. ECG changes suggest that castration may result in increased QTc, a condition associated with ventricular tachycardia, a fatal cardiac adverse event. Therefore, patients receiving hormone therapy such as $\mathrm{CAB}$ for prostate cancer should be followed up regarding cardiac consequences. Further large-scale and prospective studies may shed light on the adverse cardiac effects of the castration of patients with prostate cancer. 


\section{REFERENCES}

[1] A. Jemal, R. Siegel, E. Ward, T. Murray, J. Xu and M. J. Thun, "Cancer Statistics," CA Cancer Journal of Clinicians, Vol. 57, No. 1, 2007, pp. 43-66. doi:10.3322/canjclin.57.1.43

[2] A. Jemal, F. Bray, M. Center, J. Ferlay, E. Ward and D. Forman, "Global Cancer Statistics," CA Cancer Journal of Clinicians, Vol. 61, No. 2, 2011, pp. 69-90. doi:10.3322/caac.20107

[3] A. Karl and B. Konety, "Androgen Deprivation Therapy for Prostate Cancer: Indications, Contraindications and Possible Consequences," F1000 Medicine Reports, 2009. http://www.ncbi.nlm.nih.gov/pmc/articles/PMC2920692

[4] G. W. Chodak, T. Keane and L. Klotz, "Critical Evaluation of Hormonal Therapy for Carcinoma of the Prostate," Urology, Vol. 60, No. 2, 2002, pp. 201-208. doi:10.1016/S0090-4295(02)01677-1

[5] V. B. Shahinian, Y. F. Kuo, J. L. Freeman and J. S. Goodwin, "Risk of Fracture after Androgen Deprivation for Prostate Cancer," The New England Journal of Medicine, Vol. 352, No. 2, 2005, pp. 154-164. doi:10.1056/NEJMoa041943

[6] F. A. S. Chutz and W. K. Oh, "Neoadjuvant and Adjuvant Therapies in Prostate Cancer," Urologic Clinics of North America, Vol. 37, No. 1, 2010, pp. 97-104. doi:10.1016/j.ucl.2009.11.012

[7] C. S. Saigal, J. L. Gore, T. L. Krupski, J. Hanley, M. Schonlau and M. S. Litwin, "Androgen Deprivation Therapy Increases Cardiovascular Morbidity in Men with Prostate Cancer," Cancer, Vol. 110, No. 7, 2007, pp. 1493-1500. doi:10.1002/cncr.22933

[8] P.-C. Yang, J. Kurokawa, T. Furukawa and C. E. Clancy, "Acute Effects of Sex Steroid Hormones on Susceptibility to Cardiac Arrhythmias: A Simulation Study," PLOS Computational Biology, Vol. 6, No. 1, 2010, Article ID: e1000658.

http://www.ncbi.nlm.nih.gov/pmc/articles/PMC2813260

[9] M. F. Kalin and B. Zumoff, "Sex Hormones and Coronary Disease: A Review of the Clinical Studies," Steroids, Vol. 55, No. 8, 1990, pp. 30-52. doi:10.1016/0039-128X(90)90058-J

[10] W. Weidemann and H. Hanke, "Cardiovascular Effects of Androgens," Cardiovascular Drug Reviews, Vol. 20, No. 3, 2002, pp. 175-198. doi:10.1111/j.1527-3466.2002.tb00086.x

[11] R. W. Rockhold, "Cardiovascular Toxicity of Anabolic Steroids," Annual Review of Pharmacology and Toxicology, Vol. 33, 1993, pp. 497-520. doi:10.1146/annurev.pa.33.040193.002433

[12] P. Alexandersen, J. Haarbo, I. Byrjalsen, H. Lawaetz and C. Christiansen, "Natural Androgens Inhibit Male Atherosclerosis: A Study in Castrated, Cholesterol-Fed Rabbits," Circulation Research, Vol. 87, No. 7, 1999, pp. 813-819. doi:10.1161/01.RES.84.7.813

[13] M. T. Doğru, M. M. Başar, E. Yuvanç, V. Simşek and O. Sahin, "The Relationship between Serum Sex Steroid Levels and Heart Rate Variability Parameters in Males and the Effect of Age," Turk Kardiyol Dern Ars, Vol. 38, No.
7, 2010, pp. 459-465.

[14] T. M. Saleh, A. E. Cribb and B. J. Connell, "Role of Estrogen in Central Nuclei Mediating Stroke-Induced Changes in Autonomic Tone," Journal of Stroke and Cerebrovascular Diseases, Vol. 12, No. 9, 2003, pp. 182195. doi:10.1016/S1052-3057(03)00080-6

[15] S. Tsang, S. Wu, J. Liu and T. M. Wong, "Testosterone Protects Rat Hearts against Ischaemic Insults by Enhancing the Effects of Alpha(1)-Adrenoceptor Stimulation," British Journal of Pharmacology, Vol. 153, No. 4, 2008 , pp. 693-709. doi:10.1038/sj.bjp.0707624

[16] P. Y. Liu, A. K. Death and D. J. Handelsman, "Androgens and Cardiovascular Disease," Endocrine Reviews, Vol. 24, No. 3, 2003, pp. 313-340. doi:10.1210/er.2003-0005

[17] N. L. Keating, A. J. O’Malley and M. R. Smith, "Diabetes and Cardiovascular Disease during Androgen Deprivation Therapy for Prostate Cancer," Journal of Clinical Oncology, Vol. 24, No. 27, 2006, pp. 4448-4456. doi:10.1200/JCO.2006.06.2497

[18] K. Ezaki, M. Nakagawa, Y. Taniguchi, Y. Nagano, Y. Teshima, K. Yufu, et al., "Gender Differences in the ST Segment: Effect of Androgen-Deprivation Therapy and Possible Role of Testosterone," Circulation Journal, Vol. 74, No. 11, 2010, pp. 2448-2454. doi:10.1253/circj.CJ-10-0221

[19] S. M. Al-Khatib, N. M. LaPointe, J. M. Kramer and R. M. Califf, "What Clinicians Should Know About the QT Interval," The Journal of the American Medical Association, Vol. 289, No. 16, 2003, pp. 2120-2127. doi:10.1001/jama.289.16.2120

[20] M. Zitzmann and E. Nieschlag, "Androgen Receptor Gene CAG Repeat Length and Body Mass Index Modulate the Safety of Long-Term Intramuscular Testosterone Undecanoate Therapy in Hypogonadal Men," The Journal of Clinical Endocrinology \& Metabolism, Vol. 92, No. 10, 2007, pp. 3844-3853. doi:10.1210/jc.2007-0620

[21] S. N. Culos-Reed, J. L. Robinson, H. Lau, K. O'Connor and M. R. Keats, "Benefits of a Physical Activity Intervention for Men with Prostate Cancer," Journal of Sport \& Exercise Psychology, Vol. 25, No. 1, 2007, pp. 118-127.

[22] C. van Noord, M. Dörr, M. C. Sturkenboom, S. M. Straus, T. Reffelmann, S. B. Felix, et al., "The Association of Serum Testosterone Levels and Ventricular Repolarization," European Journal of Epidemiology, Vol. 25, No. 1, 2010, pp. 21-28. doi:10.1007/s10654-009-9406-Z

[23] G. F. Pecori, P. M. Toja, B. Filippini, J. Michailidis, M. Scacchi, M. S. Badiale, et al., "Increased Prevalence of Prolonged QT Interval in Males with Primary or Secondary Hypogonadism: A Pilot Study," International Journal of Andrology, Vol. 33, No. 1, 2010, pp. e132-e138. doi:10.1111/j.1365-2605.2009.00985.x

[24] A. Kirilmaz, E. Bolu, F. Kilicaslan, K. Erinc, M. Uzun, E. Isik, et al., "Comparison of Electrocardiographic Repolarization Patterns between Hypogonad Males and Normal Subjects," Annals of Noninvasive Electrocardiology, Vol. 8, No. 4, 2003, pp. 284-288. doi:10.1046/j.1542-474X.2003.08404.x 